\title{
Tourisme, mobilités et santé : argumentaire
}

\section{Virginie Chasles et Philippe Duhamel}

\section{OpenEdition \\ Journals}

Édition électronique

URL : http://journals.openedition.org/rfst/673

DOI : $10.4000 /$ rfst.673

ISSN : 2492-3672

Éditeur

Espaces et SOciétés (UMR 6590)

\section{Référence électronique}

Virginie Chasles et Philippe Duhamel, «Tourisme, mobilités et santé : argumentaire », Revue

francophone sur la santé et les territoires [En ligne], Tourisme, Mobilités et Santé, mis en ligne le 25 octobre 2016, consulté le 06 avril 2021. URL : http://journals.openedition.org/rfst/673 ; DOI : https:// doi.org/10.4000/rfst.673

Ce document a été généré automatiquement le 6 avril 2021.

\section{cc) (†) (-)}

La Revue francophone sur la santé et les territoires est mise à disposition selon les termes de la Licence Creative Commons Attribution - Pas d'Utilisation Commerciale - Partage dans les Mêmes Conditions 4.0 International. 


\title{
Tourisme, mobilités et santé : argumentaire
}

\author{
Virginie Chasles et Philippe Duhamel
}

1 Si l'on considère que le tourisme est "un système d'acteurs de pratiques et de lieux qui permet à chaque individu d'aller vivre temporairement ailleurs pour assurer sa recréation, alors on voit le lien qui peut exister avec la santé. En effet, faire du tourisme une activité récréative au sens de «moment de récréation » au sens où l'on peut jouer, se reposer... mais aussi « moment de reconstitution du corps et de l'esprit » (Knafou et al., 1997). Cela signifie qu'être touriste, c'est « se faire du bien ».

2 L'observation historique du développement touristique nourrit cette première idée car le tourisme est né d'une double logique : la découverte dans sa filiation au Grand Tour, et le thérapeutique dans sa filiation au thermal. Mais il y a apporté aussi ses propres logiques et innovations avec le bain de mer et les cures de soleil dès le 19ème siècle. Il y a un lien originel entre tourisme et santé. Cette relation a évolué au fil du temps et l'avènement des trois S (Sea, San and Sun) dans les années 1920 montrent un tournant où l'hédonisme prend le pas sur le thérapeutique (MIT, 2005) en "marginalisant» l'activité thermale dans son acception de «cure » et en valorisant davantage la notion de «bien-être " et de plaisir du corps : une eau chaude, un corps bronzé, un corps étendu sur une plage de sable. Pourtant, les années 1950-1970 ont été également l'âge d'or des sanatoriums et des maisons d'enfants qui ont contribué très largement à la renommée de certaines régions comme le Massif Central et certaines vallées des Alpes du nord en France (Plateau d'Assy par exemple).

3 Aujourd'hui la relation santé/tourisme est à nouveau interrogée par différentes dynamiques qui interrogent les liens qui peuvent de nouer/renouer dans la société mondiale contemporaine. D'une part, depuis une trentaine d'années, le développement de l'idée de «bien-être» est venue nourrir le catalogue des produits/séjours touristiques proposés aux personnes. Ici les maitres-mots sont la thalassothérapie, les «spa » construisant une offre de soins et de services dont l'implantation est globale et concernent de très nombreux lieux touristiques en complément leur offre ou d'autres en redynamisant leur équipement par la reconstruction/rénovation/agrandissement 
des établissements thermaux; d'autre part, cette relation entre santé et tourisme connaît depuis les années 1990 de profondes évolutions en lien avec les besoins de santé grandissants des individus (vieillissement de la population, médicalisation accrue de la société, évolution du tableau épidémiologique), les évolutions sociales et juridiques (standards esthétiques, contournement des lois nationales comme dans le cas de la Procréation Médicalement Assistée ou la thérapie cellulaire) et les stratégies de développement de certains territoires (marketing territorial, exploitation du potentiel thérapeutique d'espaces touristiques, instrumentalisation de pratiques culturelles comme le tourisme religieux).

4 L'évolution de l'offre de soin dans les territoires (au niveau national mais surtout international) et des pratiques thérapeutiques individuelles, ce que l'on appelle de manière courante mais abusive le "tourisme de santé " ou "tourisme médical " (Chasles, 2012), interrogent donc de manière très originale les liens entre "santé » et « tourisme. De nombreux travaux ont déjà montré le développement de ces produits et pratiques avec une explication très intéressante des logiques inhérentes à ces mobilités. Tout cela interroge non seulement sur l'évolution mondiale des offres de soins (préventives et curatives) mais également interpellent sur les destinations touristiques dans la mesure où de plus en plus de stations touristiques deviennent des lieux de convalescence pour les personnes ayant subi des interventions chirurgicales sérieuses voire lourdes, permettant ainsi un fonctionnement annuel de lieux marqués par la saisonnalité, forte parfois. Tout cela n'est pas sans conséquence sur les sociétés locales dans leur accès à certains équipements et soins, produisant ainsi peut-être « un cercle vertueux ».

5 L'appel à texte que nous proposons, vise à interroger cette relation historique et contemporaine entre "tourisme» et "santé ", avec une extension aux mobilités dérivées de cette relation pour montrer à la fois les mécaniques, les évolutions et les nouveautés de ce lien. L'approche est fondamentalement très ouverte et vise à faire émerger des points de vue originaux et novateurs sur des terrains et des contextes variés.

\section{BIBLIOGRAPHIE}

Anand R., Gupta S. - Medical Tourism, a growth industry, IFCAI University Press, 2008.

Bookman M.Z., Bookman K.R. - Medical tourism in developing countries, Palgrave Macmillan Publishers, 2007.

Bose J., Sumathi B. - Medical tourism, perspectives and specific country experiences, IFCAI University Press, 2008.

Chasles V. - Se déplacer pour se faire soigner : une mobilité en expansion, généralement appelée «tourisme médical », in Géoconfluences, février 2012.

Connell J. - Contemporary medical tourism: conceptualisation, culture and commodification, in Tourism Management, volume 34, February 2013, pp. 1-13. 
Connell J. - « Medical tourism: sea, sun, sand and ... surgery », in Tourism Management, $\mathrm{n}^{\circ}$ 27, pp. 1093-1100, 2006

Duhamel P. - Tourisme et temps long. Pour une généalogie des lieux touristiques. Le cas français mis en perspective. Université d'Angers, Habilitation à Diriger des Recherches, 2008.

Équipe MIT - Tourismes 1. Lieux communs, Belin, coll. Mappemonde, 2002, rééd. 2008.

Équipe MIT - Tourismes 2. Moments de lieux, Belin, coll. Mappemonde, 2005.

Équipe MIT - Tourismes 3. La Révolution durable, Belin, coll. Mappemonde, 2011.

Gesler W. M. - Bath's reputation as a healing place. In Kearns R. and Gesler W. (eds), Putting health into places: Landscape, Identity and Well-Being, Syracuse University Press. Chapter 2, pp. 17-35, 1998.

Gesler W. M. - « Lourdes: healing in a place of pilgrimage », in Health and Place, volume 2, issue 2, pp. 95-105, juin 1996.

Heng Leng C. - Medical Tourism in Malaysia : International Movement of Healthcare Consumers and the Commodification of Healthcare, Asia Research Institute, Working Paper Series $n^{\circ} 83$, janvier 2007.

Knafou R., Bruston M., Deprest F., Duhamel P., Gay J.-C. et Sacareau I. - "Une approche géographique du tourisme", l’Espace géographique, n³ 3, pp. 193-204, 1997.

Le Borgne C. - « Le tourisme médical : une nouvelle façon de se soigner », in Sève 2007/2, n¹5, pp. 47-53.

Lunt N. \& Horsfall D., Medical tourism, in Encyclopedia of Health Economics, 2014, pp. 263-270.

Mac Ready N. - « Developing countries court medical tourists », in Lancet, n 369, pp. 1849-1850, 2007.

Oppenheimer A. - « Tourisme médical, vos patients sont les bienvenus », in Courrier International, $n^{\circ} 900,2008$.

Pruthi R. - Medical tourism in India, Arise Publishers, New Delhi, 2006.

Rajagopalan S. - Health tourism, an introduction, IFCAI University Press, 2006.

Singh P. K. - Medical tourism, global outlook and Indian scenario, Kanishka publishers, 2008.

Stephano R. M. - «First Patient Surveys on Medical Tourism », in Medical Tourism Magazine, Issue 10, pp. 34-36., mai-juin 2009.

Wolfe S.M. (dir.), « Patients without borders: the emergence of medical tourism », in Health Letter, Public Citizen Health Research Group, volume 22, n 7, pp. 1-3, juillet 2006.

INDEX

Keywords : tourism, mobility, health

Mots-clés : tourisme, mobilités, santé

\section{AUTEURS}

VIRGINIE CHASLES

MCF en géographie, Université Jean Moulin Lyon 3, EA HESPER 


\section{PHILIPPE DUHAMEL}

Professeur de géographie, Directeur du département Tourisme et Loisirs, Université d'Angers, UMR 6590 Espaces et Sociétés (ESO) 